\title{
Deep Learning-Assisted TeraHertz QPSK Detection Relying on Single-Bit Quantization
}

\author{
Dongxuan He, Zhaocheng Wang, Fellow, IEEE, Tony Q. S. Quek, Fellow, IEEE, \\ Sheng Chen, Fellow, IEEE, and Lajos Hanzo, Fellow, IEEE
}

\begin{abstract}
TeraHertz (THz) wireless communication constitutes a promising technique of satisfying the ever-increasing appetite for high-rate services. However, the ultra-wide bandwidth of $\mathrm{THz}$ communications requires high-speed, high-resolution analog-todigital converters, which are hard to implement due to their high complexity and power consumption. In this paper, a deep learning-assisted $\mathrm{THz}$ receiver is designed, which relies on singlebit quantization. Specifically, the imperfections of $\mathrm{THz}$ devices, including their in-phase/quadrature-phase imbalance, phase noise and nonlinearity are investigated. The deflection ratio of the maximum-likelihood detector used by our single-bit-quantization $\mathrm{THz}$ receiver is derived, which reveals the effect of phase offset on the demodulation performance, guiding the architecture design of our proposed receiver. To combat the performance loss caused by the above-mentioned distortions, a twin-phase training strategy and a neural network based demodulator are proposed, where the phase offset of the received signal is compensated before sampling. Our simulation results demonstrate that the proposed deep learning-assisted receiver is capable of achieving a satisfactory bit error rate performance, despite the grave distortions encountered.
\end{abstract}

Index Terms-TeraHertz communication, hybrid distortion, single-bit receiver, phase compensator, deep feedforward neural network

\section{INTRODUCTION}

Given the ever-increasing data transmission demands, TeraHertz (THz) communication (from 0.1 to $10 \mathrm{THz}$ ), which provides ultra broad bandwidth reaching dozens or even hundreds of $\mathrm{GHz}$, has become a promising research area in wireless communications [1]-[4], because it is capable of supporting bandwidth-thirsty holographic video conferencing, ultra-highdefinition video transmission, fiber extender and other demanding applications [5]. Therefore, $\mathrm{THz}$ communication may be viewed as one of the key technologies in next-generation wireless communication systems [6].

This work was supported in part by National Key R\&D Program of China under Grant 2018YFB1801102, in part by Postdoctoral Science Foundation of China under Grant 2020M670332, in part by Singapore and Infocomm Media Development Authority under its Future Communications Research \& Development Programme, in part by the Engineering and Physical Sciences Research Council projects EP/P034284/1 and EP/P003990/1 (COALESCE), and in part by the European Research Council's Advanced Fellow Grant QuantCom (Grant No. 789028). (Corresponding author: Zhaocheng Wang.)

D. He and Z. Wang are with Beijing National Research Center for Information Science and Technology, Department of Electronic Engineering, Tsinghua University, Beijing 100084, China, and Z. Wang is also with Tsinghua Shenzhen International Graduate School, Shenzhen 518055, China (E-mails: dongxuan_he@mail.tsinghua.edu.cn, zcwang@tsinghua.edu.cn).

T. Q. S. Quek is with the Information Systems Technology and Design Pillar, Singapore University of Technology and Design, Singapore 487372 (e-mail: tonyquek@sutd.edu.sg)

S. Chen and L. Hanzo are with the School of Electronics and Computer Science, University of Southampton, Southampton SO17 1BJ, U.K.(E-mails: sqc@ecs.soton.ac.uk, lh@ecs.soton.ac.uk).
As the carrier frequency increases, $\mathrm{THz}$ signals tend to suffer from more serve pass-loss than their counterparts operating at lower frequencies, including both the free space path-loss and the molecular absorption loss [7]. Moreover, the imperfections of the radio frequency (RF) devices, including the in-phase/quadrature-phase (I/Q) imbalance of the RF chains [8], the nonlinearity of the power amplifier (PA) [9], and the phase noise of the local oscillator (LO), becomes more aware, imposing severe so-called hybrid distortions [10]. Moreover, given the extremely wide bandwidth, it is also hard to digitize the $\mathrm{THz}$ signals, since high-speed high-resolution analog-to-digital converters (ADCs) have a high complexity and high power consumption, which is a key challenge of THz receivers [11], [12].

Single-bit quantization, which can be realized by a fast comparator, has the potential of reducing both the power consumption and complexity [13], [14]. Therefore, singlebit ADCs have been viewed as promising techniques of facilitating ultra-high rate communications [12], [15]-[17]. For instance, Hoyos et al. [15] proposed a matched-filter based single-bit receiver for binary phase shift keying (BPSK) modulation, while Yin et al. [16] conceived an alternative digital monobit-receiver architecture for impulse radio modulation. As a further development, Wang et al. [17] took the impact of I/Q imbalances into consideration in the design of single-bit quadrature phase shift keying (QPSK) receivers. Neuhaus et al. proposed a temporal oversampling based single-bit receiver for $\mathrm{THz}$ communications, but the impact of hybrid distortions has not been considered in [12]. Moreover, Yin et al. [17] have shown that the phase offset has a significant effect on the receiver performance both in additive white Gaussian noise (AWGN) channels and multipath channels. But again, the influence of phase offset has not been studied in as much detail as the methodology of phase offset compensation in singlebit receivers. Accordingly, the state-of-the-art phase synchronization schemes designed for single-bit receivers, such as the Bayesian scheme of [19], [20], fail to perfectly compensate the phase offset under hybrid distortions. More particularly, the phase offset, which is the dominant factor in degrading the performance of single-bit receivers, cannot be adjusted, once the signals have been digitized by the single-bit ADC. As a result, the phase offset must be adjusted before sampling, which is one of the key technical challenges in single-bit $\mathrm{THz}$ receiver design.

Both the strong nonlinearities of single-bit quantization and hybrid distortions of these high-frequency devices make $\mathrm{THz}$ signals hard to process by state-of-the-art techniques. Although Singh and Madhow [18] proposed a sophisticated phase quantization technique for mitigating the I/Q phase 
TABLE I

CONTRASTING OUR CONTRibution to The Literature

\begin{tabular}{|c|c|c|c|c|c|c|}
\hline & {$[12]$} & {$[15]$} & {$[16]$} & {$[17]$} & {$[18]$} & Proposed Work \\
\hline Dual-ADC architecture & $\checkmark$ & $\checkmark$ & $\checkmark$ & $\checkmark$ & & $\checkmark$ \\
\hline Training based & & & $\checkmark$ & $\checkmark$ & $\checkmark$ & $\checkmark$ \\
\hline Phase compensation & & & & & $\checkmark$ & $\checkmark$ \\
\hline Hybrid distortion & & & & $\checkmark$ & $\checkmark$ & $\checkmark$ \\
\hline Correlated symbols & & & & & & $\checkmark$ \\
\hline Wideband communication & $\checkmark$ & $\checkmark$ & $\checkmark$ & $\checkmark$ & $\checkmark$ & $\checkmark$ \\
\hline
\end{tabular}

imbalance, their scheme required four single-bit ADCs, which increases both the complexity and cost.

\section{A. Background of Deep Learning}

Benefiting from its superior ability in handling complicated and nonlinear issues, deep learning, also known as deep neural network, has been widely regarded as a promising direction in solving intractable wireless communication problems [21]. Motivated by its promising potential, various deep-learning assisted techniques [22]-[25] have been exploited for processing the single-bit signals. For example, Balevi and Andrews [22] developed a novel deep learning based single-bit receiver for orthogonal frequency division multiplexing (OFDM), where generative supervised deep neural networks and unsupervised autoencoder detection methods are utilized for estimating the channel and detecting the signal, respectively. Balevi and Andrews [23] also transformed the design of hand-crafted channel codes into the learning of a specially designed autoencoder relying on single-bit quantization. Jeon et al. [24] utilized reinforcement learning, while Zhang et al. [25] used deep learning, respectively, for signal detection and for channel estimation in multiple-input multiple-output (MIMO) systems. In a nutshell, by relying on deep learning assisted techniques, the signals quantized by single-bit ADCs can be more beneficially processed than by conventional techniques.

In this work, deep feedforward neural network (DFNN) [26] is utilized to realize efficient single-bit receiver over $\mathrm{THz}$ channel. Specifically, a DFNN typically consists of a cascade of an input layer, $L$ hidden layers and an output layer. All the layers of the DFNN are fully connected, and the output of one layer becomes the input to its subsequent layer. Mathematically, the output $\mathrm{x}^{l}$ of the $l$-th hidden layer can be expressed as

$$
\begin{aligned}
\mathbf{z}^{l} & =\mathbf{W}_{l} \mathbf{x}^{l-1}+\mathbf{b}_{l}, \\
\mathbf{x}^{l} & =f\left(\mathbf{z}^{l}\right),
\end{aligned}
$$

where $\mathbf{W}_{l}$ and $\mathbf{b}_{l}$ are the weight matrix and the bias vector of the $l$-th layer, respectively, while $f(\cdot)$ denotes the elementwise activation function. Based on DFNN, it is capable of approximating a mapping between the received signal and the demodulation result, which facilitates our single-bit receiver.

\section{B. Contributions}

Against this background, we develop a novel deep learning assisted single-bit receiver architecture. Our main contribution$\mathrm{s}$ are boldly and explicitly contrasted to the state-of-the-art in Table I at a glance as well as in more detail below:
- We analytically analyze the receiver performance of the optimal single-bit receiver operating in $\mathrm{THz}$ channels in the face of hybrid distortions with the help of the socalled deflection ratio, which reveals the effect of phase offsets on the demodulation performance and guides the design of the single-bit $\mathrm{THz}$ receiver. Accordingly, a new phase offset difference estimator is newly developed, which is capable of facilitating the phase offset compensation before sampling, thus improving the overall detection performance.

- We design the overall architecture of a $\mathrm{THz}$ receiver, which is capable of coping with severe hybrid distortions, despite using low-resolution quantization. The receiver consists of four modules, namely the phase estimator, phase compensator, single-bit ADCs, and the demodulation network. In contrast to the state-of-the-art single-bit receiver, the phase offset is compensated before sampling for realizing reliable demodulation.

- In order to reliably detect the single-bit quantized $\mathrm{THz}$ signals, we propose a twin-phase training strategy, where the phase offset is compensated before training the neural network based demodulator. In particular, a deep learning-assisted single-bit detection methodology is proposed, which significantly improves the demodulation performance by fitting an accurate mapping between the single-bit sampling sequence and the likelihood information without relying on the distribution of each sample.

Notation: Matrices and vectors are denoted by uppercase and lowercase boldface letters. The real part is denoted by $\Re(\cdot)$, and $\mathbf{j}=\sqrt{-1}$. The expectation operator is represented by $\mathbb{E}(\cdot),(\cdot)^{\mathrm{T}}$ denotes the transpose operation, and $\sim$ denotes the equality in a distribution, while $*$ is the convolution operation. The $m \times m$ identity matrix is denoted by $\mathbf{I}_{m}, \mathbf{1}_{m}$ denotes a $1 \times m$ vector with all components one, and $[\mathbf{a}]_{i}$ denotes the $i$-th element of $\mathbf{a}$.

\section{SYSTEM MODEL}

As shown in Fig. 1, we consider an end-to-end THz communication system, where both the transmitter and receiver are equipped with a single Cassegrain antenna to provide high antenna gain [27], [28].

Gray-coding is utilized to map the information bits to the QPSK symbols. Let $s_{k}=e^{\mathrm{j} g\left(d_{k 1}, d_{k 0}\right)}$ be the $k$-th transmitted symbol corresponding to the information bits $d_{k 1}$ and $d_{k 0}$, which are equally likely to be 1 and 0 . The QPSK mapping function $g\left(d_{k 1}, d_{k 0}\right)$ is defined by $g(0,0)=\pi / 4, g(0,1)=$ $3 \pi / 4, g(1,0)=-\pi / 4$, and $g(1,1)=-3 \pi / 4$. The complex 


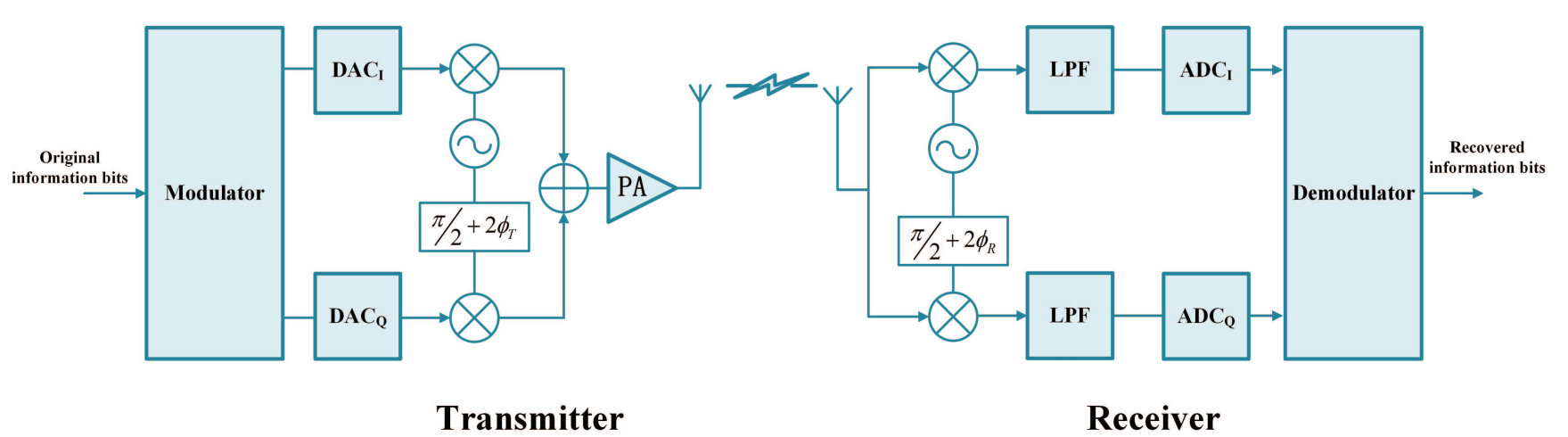

Fig. 1. System model.

baseband transmit signal can be expressed as

$$
s_{\mathrm{B}}(t)=\sum_{k=0}^{\infty} s_{k} p_{t r}\left(t-k T_{s}\right)
$$

where $p_{t r}(t)$ is the signalling pulse, and $T_{s}$ is the symbol duration. Given a perfectly I/Q-balanced carrier modulator and the perfect PA, the transmitted RF signal can be expressed as

$$
s_{\mathrm{RF}}(t)=\sqrt{2} \Re\left(e^{\mathrm{j} 2 \pi f_{c} t} s_{\mathrm{B}}(t)\right),
$$

where $f_{c}$ is the carrier frequency.

Under perfectly I/Q-balanced carrier demodulation, the received RF signal is first down-converted and then filtered by a low-pass filter (LPF) to yield the baseband received signal:

$$
r_{\mathrm{B}}(t)=\sum_{k=0}^{\infty} s_{k} e^{\mathrm{j} \Delta \theta} p_{r e f}\left(t-k T_{s}\right)+n_{\mathrm{B}}(t),
$$

where $p_{\text {ref }}(t)=p_{r}(t) * h(t) * p_{t r}$ denotes the reference signal, with $h(t)$ and $p_{r}(t)$ representing the channel impulse response (CIR) and the impulse response of the LPF, respectively. Furthermore, $\Delta \theta$ is the carrier phase offset between the transmitter and the receiver, which is unknown to the receiver, and $n_{\mathrm{B}}(t)$ is the baseband-equivalent complex Gaussian noise.

\section{A. Hybrid Distortion of $\mathrm{THz}$ Devices}

Due to the serious imperfections of $\mathrm{THz}$ devices, the signals will be distorted both at the transmitter and receiver. Let us now discuss the components of the hybrid distortion one by one.

1): Firstly, practical carrier modulation is imperfect. Specifically, the mismatch between the two RF branches imposes I/Q imbalance, which can be modeled by the following expression

$$
\mathbf{T}=\left[\begin{array}{ll}
1 & \varepsilon_{T} \sin \left(\phi_{T}\right) \\
0 & \varepsilon_{T} \cos \left(\phi_{T}\right)
\end{array}\right]
$$

where $\varepsilon_{T}$ and $\phi_{T}$ are the amplitude and phase imbalances between the I and $\mathrm{Q}$ branches, respectively. If we denote the inphase and quadrature signals of the perfect RF signal $s_{\mathrm{RF}}(t)$ as $s_{I}(t)$ and $s_{Q}(t)$, respectively, the actual inphase and quadrature signals $x_{I}(t)$ and $x_{Q}(t)$ of the carrier modulated signal are given respectively by

$$
\begin{aligned}
x_{I}(t) & =s_{I}(t)+\varepsilon_{T} \sin \left(\phi_{T}\right) s_{Q}(t), \\
x_{Q}(t) & =\varepsilon_{T} \cos \left(\phi_{T}\right) s_{Q}(t) .
\end{aligned}
$$

2): Secondly, due to the PA nonlinearity, the signal suffers from nonlinear distortion, which includes both amplitude compression and phase rotation. We adopt the dual-input nonlinear amplifier model to relate the input and output signals of the PA, which can be expressed as [29]

$$
\nabla_{L}\left[\begin{array}{c}
x_{I}(t) \\
x_{Q}(t)
\end{array}\right]=\left[\begin{array}{c}
\nabla_{I} \mathbf{x}(t) \\
\nabla_{Q} \mathbf{x}(t)
\end{array}\right]
$$

where $\mathbf{x}(t)=\left[x_{I}(t), x_{Q}(t)\right]^{T}, \nabla_{L}$ represents the overall nonlinear operator of the PA, while $\nabla_{I}$ and $\nabla_{Q}$ denote the nonlinear operators for the inphase and quadrature signals, respectively. In particular, the odd-order memoryless polynomial (MLP) model is utilized to describe the nonlinear distortion of the inphase and quadrature-phase branches, given by [10]

$$
\nabla_{i} \mathbf{x}_{i}(t)=\sum_{k=1, k \text { is odd }}^{K_{i}} \alpha_{i, k} \mathbf{1}_{k}(\underbrace{\mathbf{x}(t) \otimes \cdots \otimes \mathbf{x}(t)}_{\text {The number of } \mathbf{x}(t) \text { is } k}),
$$

where $K_{i}$ is the order of nonlinearity and $\alpha_{i, k}$ are the realvalued model parameters, for $i=I$ or $Q$. $\otimes$ denotes the Kronecker product, given by

$$
\left[\begin{array}{l}
x_{1} \\
x_{2}
\end{array}\right] \otimes\left[\begin{array}{l}
y_{1} \\
y_{2}
\end{array}\right]=\left[\begin{array}{l}
x_{1} y_{1} \\
x_{1} y_{2} \\
x_{2} y_{1} \\
x_{2} y_{2}
\end{array}\right]
$$

Taking into account the carrier modulation I/Q imbalance and PA nonlinearity, therefore, the actual transmitted RF inphase and quadrature signals can be modeled as $\nabla_{L} \mathbf{T s}_{\mathbf{R F}}(t)$ with $\mathbf{s}_{\mathrm{RF}}(t)=\left[s_{I}(t) s_{Q}(t)\right]^{\mathrm{T}}$.

3): Similarly, due to the imbalance of the two RF branches and the phase noise of the receiver's voltage-controlled oscillator (VCO), the received RF signal is further distorted during demodulation, and the distortion can be expressed as

$$
\mathbf{R}=\left[\begin{array}{ll}
1 & \varepsilon_{R} \sin \left(\phi_{R}\right) \\
0 & \varepsilon_{R} \cos \left(\phi_{R}\right)
\end{array}\right]^{\mathrm{T}}\left[\begin{array}{cc}
\cos (\theta) & -\sin (\theta) \\
\sin (\theta) & \cos (\theta)
\end{array}\right]
$$

where $\varepsilon_{R}$ and $\phi_{R}$ are the amplitude imbalance and phase imbalance between the I and Q branches at the receiver, respectively, while $\theta$ is the phase shift caused by the phase noise at 
the transmitter and the VCO of the receiver. ${ }^{1}$ Specifically, we assume that the phase noise $\theta$ follows a block-based random walk model, which will change once per transmission block ${ }^{2}$. Hence, the phase noise can be expressed as $\theta_{k+1}=\theta_{k}+\delta \theta_{k}$, where $\theta_{k}$ is the phase noise of the $k$-th block and $\delta \theta_{k}$ is the change of the phase noise between adjacent blocks, which is a Gaussian random variable with zero mean and variance $\vartheta^{2}$, i.e., $\delta \theta_{k} \sim N\left(0, \vartheta^{2}\right)$.

As a result, after demodulation and low-pass filtering, the baseband signal received over the $\mathrm{THz}$ channel under hybrid distortions can be expressed as

$$
\mathbf{r}_{\mathrm{B}}(t)=\mathbf{R}\left(\mathbf{H} \nabla_{L} \mathbf{T s}_{\mathrm{B}}(t)+\mathbf{n}_{\mathrm{B}}(t)\right),
$$

where $\mathbf{r}_{\mathrm{B}}(t)=\left[r_{\mathrm{B}_{I}}(t) r_{\mathrm{B}_{Q}}(t)\right]^{\mathrm{T}}$ with the complex baseband received signal $r_{\mathrm{B}}(t)=r_{\mathrm{B}_{I}}(t)+\mathrm{j} r_{\mathrm{B}_{Q}}(t)$, and $\mathbf{s}_{\mathrm{B}}(t)=$ $\left[s_{\mathrm{B}_{I}}(t) s_{\mathrm{B}_{Q}}(t)\right]^{\mathrm{T}}$ with the complex baseband transmit signal $s_{\mathrm{B}}(t)=s_{\mathrm{B}_{I}}(t)+\mathrm{j} s_{\mathrm{B}_{Q}}(t)$, while $\mathbf{n}_{\mathrm{B}}(t)=\left[n_{\mathrm{B}_{I}}(t) n_{\mathrm{B}_{Q}}(t)\right]^{\mathrm{T}}$ with the low-pass filtered complex channel AWGN $n_{\mathrm{B}}(t)=$ $n_{\mathrm{B}_{I}}(t)+\mathrm{j} n_{\mathrm{B}_{Q}}(t)$. Both $n_{\mathrm{B}_{I}}(t)$ and $n_{\mathrm{B}_{Q}}(t)$ have power $\sigma_{0}^{2}$. Furthermore, the overall channel response $\mathbf{H}$ can be expressed as

$$
\mathbf{H}=\alpha_{\mathrm{PL}} e^{\mathrm{j} \kappa} \mathbf{I}_{2},
$$

where $\alpha_{\mathrm{PL}}$ denotes the path loss, which includes the impact of antenna misalignment, frequency dependent loss, and frequency dispersion index, while $\kappa$ denotes the phase shift of the channel.

For notational simplification, by omitting the baseband subscript ${ }_{\mathrm{B}}$ from $\mathbf{r}_{\mathrm{B}}(t), \mathbf{s}_{\mathrm{B}}(t)$ and $\mathbf{n}_{\mathrm{B}}(t)$ as well as defining $\boldsymbol{\Sigma}=\mathbf{R H} \nabla_{L} \mathbf{T}$ and $\mathbf{n}_{r}(t)=\mathbf{R n}(t)$, (13) can be rewritten as

$$
\mathbf{r}(t)=\Sigma \mathbf{s}(t)+\mathbf{n}_{r}(t),
$$

with $\mathbf{r}(t)=\left[r_{I}(t) r_{Q}(t)\right]^{\mathrm{T}}$. Before sampling, the received signal is rotated by $\Delta^{2} \theta$ for improving the demodulation performance, where the residual phase offset can be calculated as $\Delta \theta=\theta+\kappa+\Delta^{2} \widehat{\theta}$. As a result, the received baseband signal is given by

$$
\widetilde{\mathbf{r}}(t)=\left[\begin{array}{cc}
\cos \left(\Delta^{2} \hat{\theta}\right) & -\sin \left(\Delta^{2} \widehat{\theta}\right) \\
\sin \left(\Delta^{2} \hat{\theta}\right) & \cos \left(\Delta^{2} \hat{\theta}\right)
\end{array}\right] \mathbf{r}(t),
$$

with $\widetilde{\mathbf{r}}(t)=\left[\widetilde{r}_{I}(t) \widetilde{r}_{Q}(t)\right]^{\mathrm{T}}$. Next, the received baseband signal is sampled by the single-bit ADCs, where every pulse is represented by $N_{s}$ samples, that is, the sampling period $T$ satisfies $T=T_{s} / N_{s}$. Then the $n$-th sample of the I and Q branches of the $k$-th symbol can be expressed as

$$
\begin{gathered}
r_{I, k, n}= \begin{cases}+1, & \widetilde{r}_{I}\left(k T_{s}+n T\right)>0, \\
-1, & \widetilde{r}_{I}\left(k T_{s}+n T\right) \leq 0,\end{cases} \\
r_{Q, k, n}= \begin{cases}+1, & \widetilde{r}_{Q}\left(k T_{s}+n T\right)>0, \\
-1, & \widetilde{r}_{Q}\left(k T_{s}+n T\right) \leq 0,\end{cases}
\end{gathered}
$$

\footnotetext{
${ }^{1}$ For a typical VCO, the phase noise increases with the square of the center frequency, hence it cannot be ignored for a high-frequency $\mathrm{THz}$ receiver.

${ }^{2}$ Random-walk model is commonly utilized to describe strong phase noise, whereby the phase noise varies per transmission due to the ultra-high rate of $\mathrm{THz}$ communication [30].
}

for $1 \leq n \leq N_{s}$. The single-bit sampling sequence of the $k$-th symbol can be expressed as

$$
\mathbf{r}_{k}=\left[\begin{array}{ll}
\mathbf{r}_{I_{k}}^{\mathrm{T}} & \mathbf{r}_{Q_{k}}^{\mathrm{T}}
\end{array}\right]^{\mathrm{T}},
$$

with $\mathbf{r}_{I_{k}}^{\mathrm{T}}=\left[r_{I, k, 1} \cdots r_{I, k, N_{s}}\right]$ and $\mathbf{r}_{Q_{k}}^{\mathrm{T}}=\left[r_{Q, k, 1} \cdots r_{Q, k, N_{s}}\right]$.

\section{B. Optimal Single-bit Receiver}

Since $d_{k 1}$ and $d_{k 0}$ are equally likely to be 1 and 0 , the maximum-likelihood (ML) detector is the optimal detector for the single-bit sampling sequence $\mathbf{r}_{k}$ [17]. The likelihood function of the $k$-th single-bit sampling sequence, denoted as $\Lambda\left(\mathbf{r}_{k} \mid d_{k 1}, d_{k 0}\right)$, can be expressed as

$$
\begin{aligned}
& \Lambda\left(\mathbf{r}_{k} \mid d_{k 1}, d_{k 0}\right)= \\
& \sum_{n=1}^{N_{s}}\left(\log \left(1+\left(1-2 Q\left(\widetilde{\gamma}_{I, n}^{d_{k 1}, d_{k 0}}\right)\right) r_{I, k, n}\right)\right. \\
& \left.+\log \left(1+\left(1-2 Q\left(\widetilde{\gamma}_{Q, n}^{d_{k 1}, d_{k 0}}\right)\right) r_{Q, k, n}\right)\right)-2 N_{s} \log (2),
\end{aligned}
$$

where the $\mathrm{Q}$ function $Q(\cdot)$ is given by $Q(x)=$ $\frac{1}{\sqrt{2 \pi}} \int_{x}^{\infty} e^{-t^{2} / 2} d t$ and we have

$\widetilde{\gamma}_{I, n}^{d_{k 1}, d_{k 0}}=\frac{1}{\sigma_{0}}\left(\nabla_{I}\left(\mathbf{x}_{n}^{d_{k 1}, d_{k 0}}\right) \cos \Delta \theta-\nabla_{Q}\left(\mathbf{x}_{n}^{d_{k 1}, d_{k 0}}\right) \sin \Delta \theta\right)$,

$$
\begin{aligned}
\widetilde{\gamma}_{Q, n}^{d_{k 1}, d_{k 0}}= & \frac{1}{\sigma_{0}}\left(\nabla_{I}\left(\mathbf{x}_{n}^{d_{k 1}, d_{k 0}}\right) \varepsilon_{R} \sin \left(\phi_{R}+\Delta \theta\right)\right. \\
& \left.+\varepsilon_{R} \cos \left(\phi_{R}+\Delta \theta\right) \nabla_{Q}\left(\mathbf{x}_{n}^{d_{k 1}, d_{k 0}}\right)\right),
\end{aligned}
$$

with

$$
\begin{aligned}
& \mathbf{x}_{n}^{d_{k 1}, d_{k 0}}= \\
& {\left[\begin{array}{c}
\left(\left(1-2 d_{k 0}\right)+\varepsilon_{T} \sin \phi_{T}\left(1-2 d_{k 1}\right)\right) p_{r e f}\left(n T_{s} / N_{s}\right) \\
\varepsilon_{T} \cos \phi_{T}\left(1-2 d_{k 1}\right) p_{r e f}\left(n T_{s} / N_{s}\right)
\end{array}\right] .}
\end{aligned}
$$

When the distorted received symbol $\mathbf{r}_{k}$ is obtained, it is demodulated according to the likelihood function $\Lambda\left(\mathbf{r}_{k} \mid d_{k 1}, d_{k 0}\right)$ of the conventional ML demapper as

$$
\left(\widetilde{d}_{k 1}^{\text {opt }}, \widetilde{d}_{k 0}^{\text {opt }}\right)=\arg \max _{d_{k 1}, d_{k 0} \in\{0,1\}} \Lambda\left(\mathbf{r}_{k} \mid d_{k 1}, d_{k 0}\right) .
$$

To evaluate the performance of the ML receiver, the deflection ratio under QPSK modulation relying on single-bit sampling is defined by [31]

$$
D=\frac{\left(\mathbb{E}\left(\widetilde{\lambda}_{k} \mid d_{k 1}=1, d_{k 0}=1\right)-\mathbb{E}\left(\tilde{\lambda}_{k} \mid d_{k 1}=1, d_{k 0}=0\right)\right)^{2}}{\operatorname{Var}\left(\widetilde{\lambda}_{k}\right)},
$$

where $\widetilde{\lambda}_{k}=\Lambda\left(\mathbf{r}_{k} \mid d_{k 1}=1, d_{k 0}=1\right)$ is the decision statistic of the ML detector and $\operatorname{Var}\left(\lambda_{k}\right)$ denotes the variance of $\widetilde{\lambda}_{k}$. The higher $D$ is the better demodulation performance.

After some manipulations, the deflection ratio of the optimal single-bit $\mathrm{THz}$ receiver, which depends on the residual phase offset $\Delta \theta$ and $N_{s}$, can be derived as shown in (26), with 


$$
D\left(\Delta \theta, N_{s}\right)=\frac{\left(\sum_{n=1}^{N_{s}}\left(\log \left(\frac{\varpi_{I, n}^{1,1}}{\varpi_{I, n}^{1,0}}\right) \varpi_{I, n}^{1,1}+\log \left(\frac{\omega_{I, n}^{1,1}}{\omega_{I, n}^{1,0}}\right) \omega_{I, n}^{1,1}+\log \left(\frac{\varpi_{Q, n}^{1,1}}{\omega_{Q, n}^{1,0}}\right) \varpi_{Q, n}^{1,1}+\log \left(\frac{\omega_{Q, n}^{1,1}}{\varpi_{Q, n}^{1,0}}\right) \omega_{Q, n}^{1,1}\right)\right)^{2}}{\sum_{n=1}^{N_{s}}\left(\omega_{I, n}^{1,1} \varpi_{I, n}^{1,1} \log ^{2}\left(\frac{\varpi_{I, n}^{1,1}}{\omega_{I, n}^{1,1}}\right)+\omega_{Q, n}^{1,1} \varpi_{Q, n}^{1,1} \log ^{2}\left(\frac{\varpi_{Q, n}^{1,1}}{\omega_{Q, n}^{1,1}}\right)\right)} .
$$

$\omega_{I, n}^{d_{k 1}, d_{k 0}}=Q\left(\widetilde{\gamma}_{I, n}^{d_{k 1}, d_{k 0}}\right), \varpi_{I, n}^{d_{k 1}, d_{k 0}}=1-Q\left(\widetilde{\gamma}_{I, n}^{d_{k 1}, d_{k 0}}\right)$,
$\omega_{Q, n}^{d_{k 1}, d_{k 0}}=Q\left(\widetilde{\gamma}_{Q, n}^{d_{k 1}, d_{k 0}}\right)$, and $\varpi_{Q, n}^{d_{k 1}, d_{k 0}}=1-Q\left(\widetilde{\gamma}_{Q, n}^{d_{k 1}, d_{k 0}}\right)$.
Proof: See Appendix A.

\section{AnAlysis of Phase Offset}

Observe from (26) that the performance of the optimal ML receiver depends on both the weight tuple $\mathbf{w}=$ $\left(\omega_{I, n}^{d_{k 1}, d_{k 0}}, \varpi_{I, n}^{d_{k 1}, d_{k 0}}, \omega_{Q, n}^{d_{k 1}, d_{k 0}}, \varpi_{Q, n}^{d_{k 1}, d_{k 0}}\right)$ and $N_{s}$, where the weight tuple $\mathbf{w}$ is determined by the phase offset $\Delta \theta$. As a result, the phase offset is the dominant factor determining the performance of the single-bit $\mathrm{THz}$ receiver. Therefore, in this section, we first investigate the impact of the phase offset on the demodulation performance and then propose a phase compensation method to adjust the phase offset of the received baseband signal $\mathbf{r}(t)$ in order to enhance the achievable demodulation performance.

\section{A. Effects of Phase Offset}

To investigate the effects of the phase offset $\Delta \theta$ on the demodulation performance, the deflection ratio of (26) encountered in the $\mathrm{THz}$ channel at $E_{b} / N_{0}=5 \mathrm{~dB}$ with the oversampling ratio $N_{s} \in\{1,5,10,20\}$ is plotted in Fig. 2 (a) as a function of $\Delta \theta$, where $E_{b}$ denotes the transmit energy per bit and $N_{0}$ is the power spectral density of the channel noise. Specifically, the distortion parameters of $\mathrm{THz}$ channel are $\varepsilon_{T}=\varepsilon_{R}=1.2, \phi_{T}=\phi_{R}=2^{\circ}$, and the parameters of the dual-input nonlinear PA model are $K_{I}=5$ in conjunction with $\alpha_{I, 1}=1.521, \alpha_{I, 3}=-0.5626, \alpha_{I, 5}=0.0402$, and $K_{Q}=7$ along with $\alpha_{Q, 1}=2.138, \alpha_{Q, 3}=-2.749, \alpha_{Q, 5}=2.787$, $\alpha_{Q, 7}=-1.179$. For comparison, we also plot the deflection ratio curve for the AWGN channel at $E_{b} / N_{0}=5 \mathrm{~dB}$ with $N_{s} \in\{1,5,10,20\}$ in Fig. $2(\mathrm{~b})$, where $\varepsilon_{T}=\varepsilon_{R}=1$, $\phi_{T}=\phi_{R}=0^{\circ}$, and the parameters of the dual-input nonlinear PA model are $K_{I}=1$ in conjunction with $\alpha_{I, 1}=1$ and $K_{Q}=1$ along with $\alpha_{Q, 1}=1$. Three observations can be drawn from Fig. 2.

Firstly, it can be seen from Fig. 2 (b) that the deflection ratio of the AWGN channel is maximized when the phase offset is $\Delta \theta=0$, and the deflection ratio decreases significantly, when $\Delta \theta$ deviates from 0 . This is because for transmission over the AWGN channel the deflection ratio decreases significantly, when the amplitude difference between the I and Q branches is large. However, in contrast to the AWGN channel, the magnitude of the received constellation points of the signals is time-variant in the $\mathrm{THz}$ channel. As a result, the optimal phase offset to attain the maximum deflection ratio for the $\mathrm{THz}$ channel is not 0 . As can be seen from Fig. 2 (a), the deflection ratio for the $\mathrm{THz}$ channel simulated reaches the maximal value

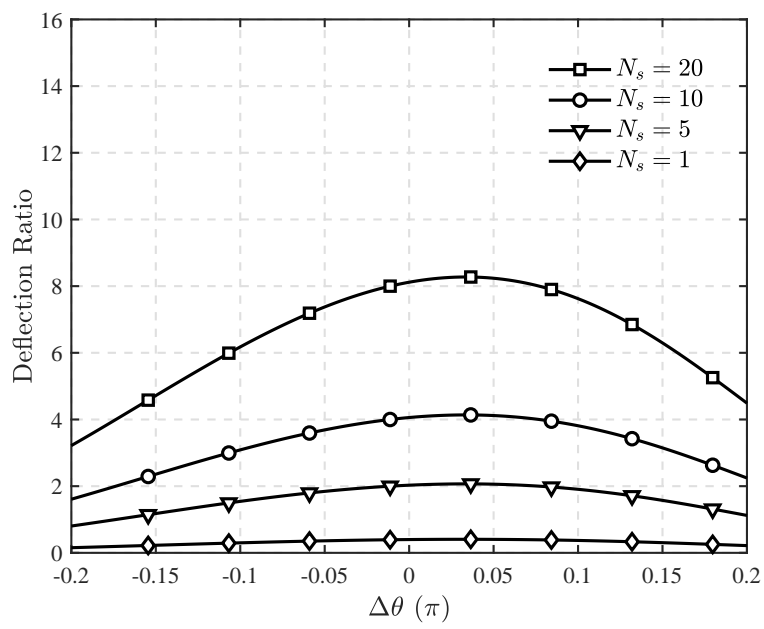

(a) $\mathrm{THz}$ channel

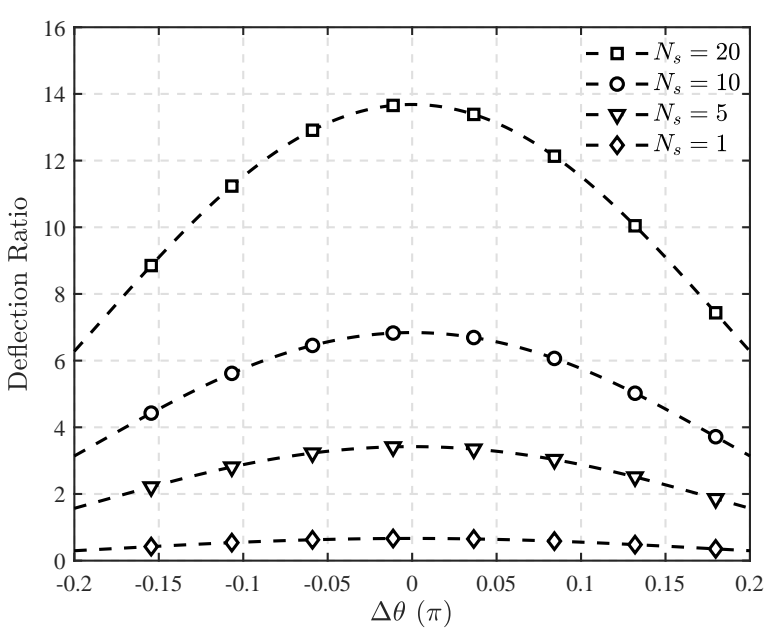

(b) AWGN channel

Fig. 2. Deflection ratio versus phase offset parameterized by oversampling ratio $N_{s}$.

when $\Delta \theta$ is close to $0.04 \pi$, and the deflection ratio decrease significantly, when $\Delta \theta$ is different from $0.04 \pi$.

Secondly, at the same $E_{b} / N_{0}$ and $N_{s}$, the deflection ratio of the THz channel is lower than that of the AWGN channel, explicitly reflecting the adverse effect of hybrid $\mathrm{THz}$ distortions.

Thirdly, the deflection ratio increases with the increase of $N_{s}$, which demonstrates that the demodulation performance can be improved by increasing the oversampling ratio $N_{s}$. Nevertheless, a higher oversampling ratio increases the device cost and power consumption. Hence, it is always important to realize an appropriate performance versus cost trade-off in practice. 


\section{B. Phase Compensation}

The above analysis demonstrates that there exists an optimal phase offset $\Delta \theta^{\star}$ that optimizes the demodulation performance, that is, the deflection ratio is maximized. However, due to the random nature of both the phase noise and the $\mathrm{THz}$ channel, the actual phase offset $\Delta \theta$ of the THz channel is unknown and different from the optimal phase offset $\Delta \theta^{\star}$. Therefore, it is necessary to compensate for the actual phase offset, i.e., adjusting $\Delta \theta$ into $\Delta \theta^{\star}$, in order to attain the optimal demodulation performance.

Note that $\Delta \theta^{\star}$ can always be estimated. This is because although the weight tuple $\mathbf{w}$ is unknown, it can be estimated by utilizing a phase offset training sequence according to

$$
\begin{aligned}
& \widehat{\omega}_{I, n}^{d_{k 1}, d_{k 0}}=\frac{1}{2}-\frac{1}{2 N_{t}^{d_{k 1}, d_{k 0}}} \sum_{k=1}^{N_{t}^{d_{k 1}, d_{k 0}}} r_{I, k, n}, \\
& \widehat{\omega}_{Q, n}^{d_{k 1}, d_{k 0}}=\frac{1}{2}-\frac{1}{2 N_{t}^{d_{k 1}, d_{k 0}}} \sum_{k=1}^{N_{t}^{d_{k 1}, d_{k 0}}} r_{Q, k, n}, \\
& \widehat{\varpi}_{I, n}^{d_{k 1}, d_{k 0}}=\frac{1}{2}+\frac{1}{2 N_{t}^{d_{k 1}, d_{k 0}}} \sum_{k=1}^{N_{t}^{d_{k 1}, d_{k 0}}} r_{I, k, n}, \\
& \widehat{\varpi}_{Q, n}^{d_{k 1}, d_{k 0}}=\frac{1}{2}+\frac{1}{2 N_{t}^{d_{k 1}, d_{k 0}}} \sum_{k=1}^{N_{t}^{d_{k 1}, d_{k 0}}} r_{Q, k, n},
\end{aligned}
$$

where $N_{t}^{d_{k 1}, d_{k 0}}$ is the length of the sequence in terms of the received symbols $\left(d_{k 1}, d_{k 0}\right)$. Substituting the estimated weight tuple $\widehat{\mathbf{w}}=\left(\widehat{\omega}_{I, n}^{d_{k 1}, d_{k 0}}, \widehat{\omega}_{Q, n}^{d_{k 1}, d_{k 0}}, \widehat{\varpi}_{I, n}^{d_{k 1}, d_{k 0}}, \widehat{\varpi}_{Q, n}^{d_{k 1}, d_{k 0}}\right)$ into (26), the estimated deflection ratio $\widehat{D}\left(\Delta \widetilde{\theta}, N_{s}\right)$ of the optimal ML receiver with the phase offset $\Delta \widetilde{\theta}$ can be obtained without relying on the knowledge of the channel information. An estimate of the optimal phase offset can then be obtained as the solution of the following optimization

$$
\Delta \widehat{\theta}^{\star}=\arg \max _{\Delta \widetilde{\theta}} \widehat{D}\left(\Delta \widetilde{\theta}, N_{s}\right) .
$$

However, what the phase compensation needs is the difference between the optimal phase offset $\Delta \theta^{\star}$ and the unknown phase offset $\Delta \theta$ of the received signal, which is denoted as

$$
\Delta^{2} \theta=\Delta \theta^{\star}-\Delta \theta \text {. }
$$

We propose an algorithm to approximately estimate $\Delta^{2} \theta$. Specifically, the phase offset training sequence is divided into $N_{p}$ subsequences, where the length of each subsequence is $N_{t}$. An initial $\Delta^{2} \widetilde{\theta}_{0}=0$ is set, an initial estimated deflection ratio $\widehat{D}_{0}=0$ is assumed, and a step size $\alpha_{\theta}$ for adjusting the phase offset is provided. The $t$-th subsequence is rotated by $\Delta^{2} \widetilde{\theta}_{t}$ before sampled by the receiver, where $\Delta^{2} \widetilde{\theta}_{t}=\Delta^{2} \widetilde{\theta}_{t-1}+\alpha_{\theta}$. Substituting the sampled $t$-th subsequence into (27), the estimated weight tuple $\widehat{\mathrm{w}}$ is obtained, and the deflection ratio $\widehat{D}_{t}$ can be calculated. If $\widehat{D}_{t}>\widehat{D}_{t-1}$, it indicates that $\Delta^{2} \widetilde{\theta}_{t}$ is adjusted in a correct direction (deflection ratio increasing) from $\Delta^{2} \widetilde{\theta}_{t-1}$ by step size $\alpha_{\theta}$. Therefore, to continue this direction, $\Delta^{2} \widetilde{\theta}_{t+1}$ should also be adjusted by $\alpha_{\theta}$ from $\Delta^{2} \widetilde{\theta}_{t}$. Otherwise, $\Delta^{2} \widetilde{\theta}_{t+1}$ needs to be adjusted to the opposite direction by $-\alpha_{\theta}$ $\overline{\text { Algorithm } 1 \text { Estimate the difference between optimal phase }}$ offset and actual phase offset $\Delta^{2} \theta$

1: Set initial estimated $\Delta^{2} \widetilde{\theta}_{0}=0$, step size to $\alpha_{\theta}$, and initial estimated deflection ratio $\widehat{D}_{0}=0$.

2: for $t=1$ to $N_{p}$ do

3: $\quad$ Set $\Delta^{2} \widetilde{\theta}_{t}=\Delta^{2} \widetilde{\theta}_{t-1}+\alpha_{\theta}$.

4: Rotate the $t$-th phase offset training subsequence by $\Delta^{2} \widetilde{\theta}_{t}$.

5: $\quad$ Sample the $t$-th phase offset training subsequence.

6: $\quad$ Calculate $\widehat{\mathbf{w}}=\left(\widehat{\omega}_{I, n}^{d_{k 1}, d_{k 0}}, \widehat{\omega}_{Q, n}^{d_{k 1}, d_{k 0}}, \widehat{\varpi}_{I, n}^{d_{k 1}, d_{k 0}}, \widehat{\varpi}_{Q, n}^{d_{k 1}, d_{k 0}}\right)$ according to (27).

7: Substituting $\widehat{\mathbf{w}}$ into (26) to obtain the estimated deflection ratio $\widehat{D}_{t}$.

8: $\quad$ if $\widehat{D}_{t}>\widehat{D}_{t-1}$ then

9: $\quad$ Set $\alpha_{\theta}=\alpha_{\theta}$.

10: $\quad$ else

11: $\quad$ Set $\alpha_{\theta}=-\alpha_{\theta}$.

12: end if

13: end for

14: Calculate $\Delta^{2} \widehat{\theta}=\frac{1}{N_{p}-N_{p, i}} \sum_{t=N_{p, i}+1}^{N_{p}} \Delta^{2} \widetilde{\theta}_{t}$

from $\Delta^{2} \widetilde{\theta}_{t}$. Clearly, the first a few $\Delta^{2} \widetilde{\theta}_{t}$ are far from the true $\Delta^{2} \theta$, and it takes some iterations, say $t=N_{p, i}$, for $\Delta^{2} \widetilde{\theta}_{t}$ to converge. Also owing to the difference between $\widehat{\mathbf{w}}$ and $\mathbf{w}$, the estimated deflection ratio is inaccurate. As a result, $\Delta^{2} \widetilde{\theta}_{t}$ will naturally fluctuate. To achieve an accurate estimate, the average value of the estimated $\Delta^{2} \widetilde{\theta}_{t}$ over $N_{p, i} \leq t \leq N_{p}$ is used as the estimate of $\Delta^{2} \theta$. Algorithm 1 summarizes this proposed estimator.

As $\Delta^{2} \widehat{\theta}$ obtained by Algorithm $\mathbf{1}$ is an estimate of the difference between the optimal phase offset and actual phase offset, the received baseband signal $\mathbf{r}(t)$ can be compensated for with $\Delta^{2} \widehat{\theta}$, and how this is achieved will be elaborated further in the next section. Appropriate values for $N_{p}, N_{t}$, $N_{p, i}$ and $\alpha_{\theta}$ will be investigated in Subsection V-C.

\section{LEARNING-AsSisted SINGLE-BIT RECEIVER}

In this section, we detail the proposed deep learning-assisted single-bit $\mathrm{THz}$ receiver.

\section{A. Single-bit Receiver Architecture}

Fig. 3 depicts the overall architecture of our proposed deep learning-assisted single-bit THz QPSK receiver. In contrast to the conventional receiver shown of Fig. 1, which collects the received signal by a pair of ADCs and demodulates the sampling sequence directly, the phase offset is first compensated by our proposed receiver before sampling and the single-bit sampled sequence is demodulated by a deep learning based demodulation network. Hence, our proposed single-bit receiver consists of a phase estimator, a phase compensator, two singlebit ADCs for the I an Q components, and a deep learning based demodulation network. The function of each module is now summarized. 


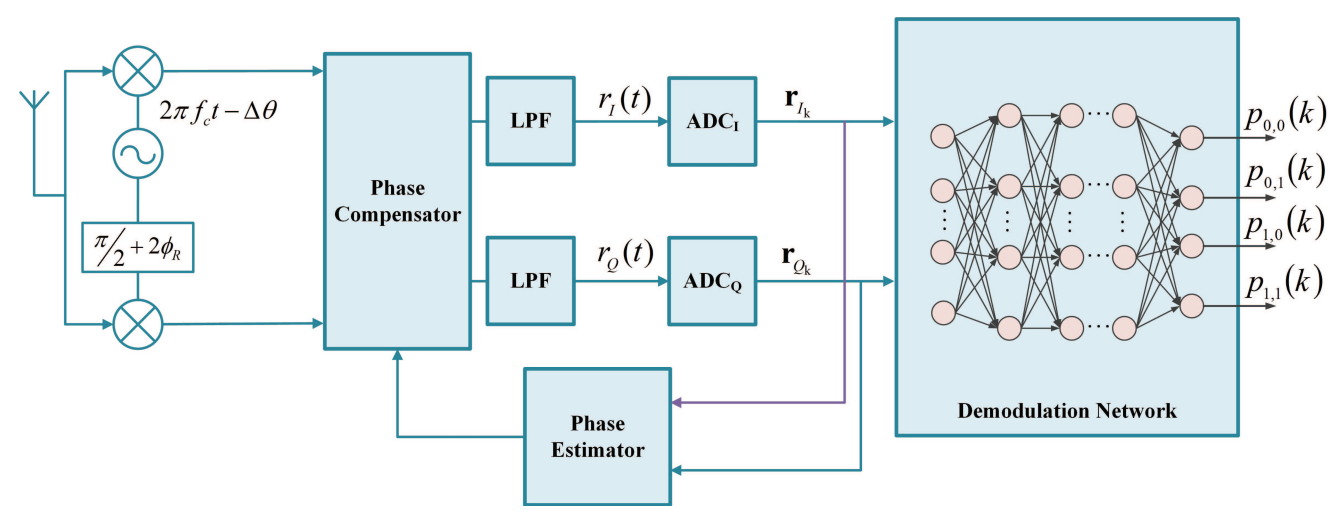

Fig. 3. The overall architecture of our proposed deep learning-assisted single-bit THz receiver.

1) Phase estimator: The phase estimator estimates the difference between the optimal phase offset and the actual phase offset of the received signal, $\Delta^{2} \theta$, as detailed in Subsection III-B. This task is very challenging as it is implicitly relying on the cost function value only.

2) Phase compensator: The phase compensator is utilized to rotate the THz signal according to the feedback $\Delta^{2} \widehat{\theta}$ from the phase estimator. Typically, the rotation operation can be realized by the local oscillator (LO), where the phase offset can be directly controlled [32].

3) Single-bit ADCs: The two single-bit ADCs are utilized for converting the I and $\mathrm{Q}$ components of the analog $\mathrm{THz}$ signals $\mathbf{r}(t)$ into two single-bit sampled sequences $\mathbf{r}_{I_{k}}$ and $\mathbf{r}_{Q_{k}}$ with the oversampling ratio of $N_{s}$.

4) Demodulation network: The demodulation network is utilized for demodulating the received single-bit sampled sequences of the $\mathrm{THz}$ signals. Note that given the hybrid distortions caused by the THz transceiver, $\Lambda\left(\mathbf{r}_{k} \mid d_{k 1}, d_{k 0}\right)$ cannot be characterized analytically. As a result, reliably detecting the THz symbols digitized by single-bit ADCs is quite a challenge. To tackle this problem, we propose a deep learning-assisted single-bit $\mathrm{THz}$ receiver that is capable of efficiently solving the ML demapper (24).

\section{B. Deep Learning Based Demodulation}

To demodulate the phase-compensated single-bit sampled sequence $\left\{\mathbf{r}_{k}\right\}$, a DFNN having $L$ hidden layers is adopted as the demodulation network, which is capable of approximating any measurable function at any desired degree of accuracy [26]. The input layer feeds the $\left(m_{0}=2 N_{s}\right)$-dimensional input $\mathbf{r}_{k}$ into the DFNN, and the signal propagates through the network layer by layer until it reaches the output layer. The number of the neurons in the $l$-th hidden layer is denoted as $m_{l}$ for $1 \leq l \leq L$. The dimension of the output layer of the DFNN is equal to the size of modulation alphabet, i.e., the output layer has $m_{\mathrm{o}}=4$ neurons, and the likelihoods of $\mathbf{r}_{k}$ belonging to the four constellation points are the demodulation output.

For demodulating the $k$-th symbol, denote the input to the 1st hidden layer as $\mathbf{x}_{k}^{0}=\mathbf{r}_{k} \in \mathbb{R}^{m_{0} \times 1}$. Specifically, the sigmoid function [26] is used in the hidden layers as our activation function, which is given by

$$
f(z)=\frac{1}{1+e^{-z}} .
$$

As the demodulation results of our deep network are the probabilities of the $k$-th symbol belonging to the QPSK constellation points, the softmax function [26] is selected as the activation function of the output layer. Specifically, based on the activation $\mathbf{z}_{k}^{o} \in \mathbb{R}^{4 \times 1}$ of the output layer, where $\mathbf{z}_{k}^{\mathrm{o}}=\mathbf{W}_{L+1} \mathbf{x}_{k}^{L}+\mathbf{b}_{L+1}$ with the output layer's weight matrix $\mathbf{W}_{L+1} \in \mathbb{R}^{4 \times m_{L}}$ and bias vector $\mathbf{b}_{L+1} \in \mathbb{R}^{4 \times 1}$, the output vector $\mathbf{p}_{k} \in \mathbb{R}^{4 \times 1}$ of the output layer is specified by

$$
\begin{aligned}
p_{i, j}(k) & =\left[\mathbf{p}_{k}\right]_{2 i+j+1}=\operatorname{softmax}\left(\left[\mathbf{z}_{k}^{\mathrm{o}}\right]_{2 i+j+1}\right) \\
& =\frac{\exp \left(\left[\mathbf{z}_{k}^{\mathrm{o}}\right]_{2 i+j+1}\right)}{\sum_{i^{\prime}=0}^{1} \sum_{j^{\prime}=0}^{1} \exp \left(\left[\mathbf{z}_{k}^{\mathrm{o}}\right]_{2 i^{\prime}+j^{\prime}+1}\right)},
\end{aligned}
$$

for $i, j \in\{0,1\}$, where $p_{i, j}(k)$ defines the probability that the single-bit sampling sequence $\mathbf{r}_{k}$ belongs to $\left(d_{k 1}, d_{k 0}\right)=(i, j)$. As a result, the index $\left(\hat{i}^{\star}, \widehat{j}^{\star}\right)$ of the element of $\mathbf{p}_{k}$ having the highest probability, i.e.,

$$
\left(\hat{i}^{\star}, \hat{j}^{\star}\right)=\arg \max _{i, j \in\{0,1\}} p_{i, j}(k),
$$

is the ML estimate of the information bits of the single-bit sampled sequence $\mathbf{r}_{k}$.

Collect all the parameters of the DFNN as $\mathbf{W}=$ $\left\{\mathbf{W}_{1}, \mathbf{b}_{1}, \cdots, \mathbf{W}_{L}, \mathbf{b}_{L}, \mathbf{W}_{L+1}, \mathbf{b}_{L+1}\right\}$, and denote the overall nonlinear mapping of the DFNN as $g(\cdot ; \mathbf{W})$, which links the single-bit sampling received sequence $\mathbf{r}$ to the demodulation likelihood information $\mathbf{p}=\left[\begin{array}{llll}p_{0,0} & p_{0,1} & p_{1,0} & p_{1,1}\end{array}\right]^{\mathrm{T}}$ :

$$
\mathbf{p}=g(\mathbf{r} ; \mathbf{W}) \text {. }
$$

To optimize the performance of learning-assisted demodulation, the task is to determine $\mathbf{W}$ by training the DFNN for ensuring $\mathbf{p}$ approaches $\mathbf{p}^{\star}$, where $\mathbf{p}^{\star}=\left[\begin{array}{llll}p_{0,0}^{\star} & p_{0,1}^{\star} & p_{1,0}^{\star} & p_{1,1}^{\star}\end{array}\right]^{\mathrm{T}}$ represents the actual probability of the transmit symbol for the single-bit sampling received sequence. Here, we adopt the cross-entropy as the learning performance metric, given by

$$
J(\mathbf{W})=-\mathbb{E}_{\mathbf{r}}\left(\sum_{i=0}^{1} \sum_{j=0}^{1} p_{i, j}^{\star} \log p_{i, j}\right) .
$$




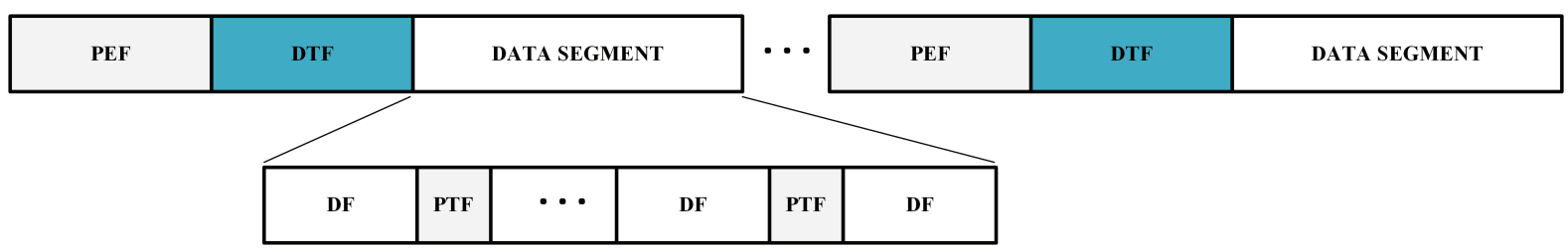

Fig. 4. The proposed transmission frame structure of $\mathrm{THz}$ communication system.

However, explicit $\mathbf{p}^{\star}$ is hard to obtain. To address this issue, we construct an empirical probability vector $\mathbf{p}^{\mathrm{e}}=$ $\left[\begin{array}{llll}p_{0,0}^{\mathrm{e}} & p_{0,1}^{\mathrm{e}} & p_{1,0}^{\mathrm{e}} & p_{1,1}^{\mathrm{e}}\end{array}\right]^{\mathrm{T}}$. Specifically, $p_{i, j}^{\mathrm{e}}=1$ and all the other three elements of $\mathbf{p}^{\mathrm{e}}$ are zero, when the $(2 i+j+1)$-th constellation symbol point is transmitted, where $i, j \in\{0,1\}$. Since $\mathbb{E}\left(\mathbf{p}^{\mathbf{e}}\right)$ is close to $\mathbf{p}^{\star}$, we use $\mathbf{p}^{\mathbf{e}}$ to replace the true target $\mathbf{p}^{\star}$. In practice, a block of training data $\left\{\mathbf{r}_{k}, \mathbf{p}_{k}^{\mathrm{e}}\right\}_{k=1}^{N_{d}}$ is used to train the network by minimizing the empirical cross entropy, given by

$$
J_{\mathrm{e}}(\mathbf{W})=-\frac{1}{N_{d}} \sum_{k=1}^{N_{d}}\left(\sum_{i=0}^{1} \sum_{j=0}^{1} p_{i, j}^{\mathrm{e}}(k) \log p_{i, j}(k)\right) .
$$

Empirical cross entropy is commonly utilized in the study of the DFNN for multi-classification problems, also called onehot code label, which has desired convergence properties and accuracy for classification tasks [26].

\section{Twin-Phase Training Strategy}

To realize the expected function of each module, a twinphase training strategy is utilized to train the single-bit receiver. In the first step, the phase estimator is trained for facilitating the optimal phase offset. In the second step, the DFNN learns the mapping between the phase-offset compensated single-bit sampled sequence and the demodulation result. An advantage of this training policy is that the adverse effect of phase noise can be eliminated, which brings significant performance improvements of the single-bit receiver. To implement this twin-phase training, the transmission frame structure of Fig. 4 is designed, which is composed of the phase estimation field (PEF), the DFNN training field (DTF) and the data segment. In particular, the data segment consists of several data fields (DF) and multiple phase tracking fields (PTF).

Estimating phase offset difference: The PEF is first transmitted to search for the optimal phase offset difference, and the process of estimating this phase offset difference is detailed in Subsection III-B. The length of PEF is $N_{p} N_{t}$, where $N_{t}=N_{t}^{(1,1)}+N_{t}^{(1,0)}$ in which $N_{t}^{(1,1)}$ and $N_{t}^{(1,0)}$ are the numbers of symbols corresponding to the bits $(1,1)$ and $(1,0)$, respectively, in the transmitted training subsequnece. We choose $N_{t}^{(1,1)}=N_{t}^{(1,0)}$ to ensure that equal numbers of the bit patters $(1,1)$ and $(1,0)$ are transmitted. This in turn ensures a balanced and more accurate estimation of the statistics $\frac{\varpi_{I, n}^{1,1}}{\varpi_{I, n}^{1,0},}$, $\frac{\omega_{I, n}^{1,1}}{\omega_{1, n}^{1,0}}, \frac{\varpi_{Q, n}^{1,1}}{\omega_{Q, n}^{1,0}}$ and $\frac{\omega_{Q, n}^{1,1}}{\varpi_{Q, n}^{1,0}}$ in (26). The estimated optimal phase offset difference is then fed to the phase compensator, where all the received symbols, including the DTF and data segment, are rotated accordingly before sampling by the single-bit ADCs.
Training demodulation network: Next, the DTF is transmitted to train the demodulation network, which consists of the DFNN training samples. In particular, each training sample includes a phase-offset-compensated single-bit sampled sequence $\mathbf{r}_{k}$ labeled by its corresponding information bits $\left(d_{k 1}, d_{k 0}\right)$. From $\left(d_{k 1}, d_{k 0}\right)$, the corresponding empirical probability target $\mathbf{p}_{k}^{\mathrm{e}}$ is constructed. Let $N_{d}$ be the length of the DTF. The receiver collects the training dataset $\mathcal{S}=$ $\left\{\mathbf{r}_{k}, \mathbf{p}_{k}^{\mathrm{e}}\right\}_{k=1}^{N_{d}}$ to train the DFNN by minimizing the cost function (35) using the scaled conjugate gradient optimizer, which is a well-known fast and efficient optimization algorithm for large-scale optimization problems [33].

After training, the DFNN can be used for demodulating the received single-bit sampled sequence during data transmission. Specifically, during data transmission, each received sequence $\mathbf{r}_{k}$ is imported into the network. Then the output of the DFNN provides the specific category or bit pattern estimate $\left(\widehat{d}_{k 1}^{\star}=\widehat{i}^{\star}, \widehat{d}_{k 0}^{\star}=\widehat{j}^{\star}\right)$ of the current received sequence according to (32).

The overall computational complexity of the proposed receiver is dominated by the training process of the DFNN. Specifically, the scaled conjugate gradient algorithm for training the DFNN has a complexity per iteration on the order of $\mathrm{O}\left(N_{\text {tot }}^{2}\right)$ [34], where $N_{\text {tot }}$ is the total number of adaptive parameters in the DFNN, given by

$$
N_{\text {tot }}=\left(2 N_{s}+1\right) m_{1}+\sum_{l=2}^{L}\left(m_{l-1}+1\right) m_{l}+\left(m_{L}+1\right) 4 .
$$

Tracking phase offset difference: Naturally, the phase shift is time-variant, and hence the PTF is inserted into the data segment for tracking the time-variant phase offset difference to mitigate the effect of the phase noise. This is achieved by the same phase offset difference estimator. The adjusted phase offset difference is used by the phase compensator to rotate the subsequent received data segment before sampling by the single-bit ADCs for demodulation.

\section{Numerical Analysis}

This section presents numerical results for characterizing the proposed single-bit $\mathrm{THz}$ receiver, including the performance of phase compensation and deep learning based demodulation. All the symbols are generated by the system model described in Section II. 


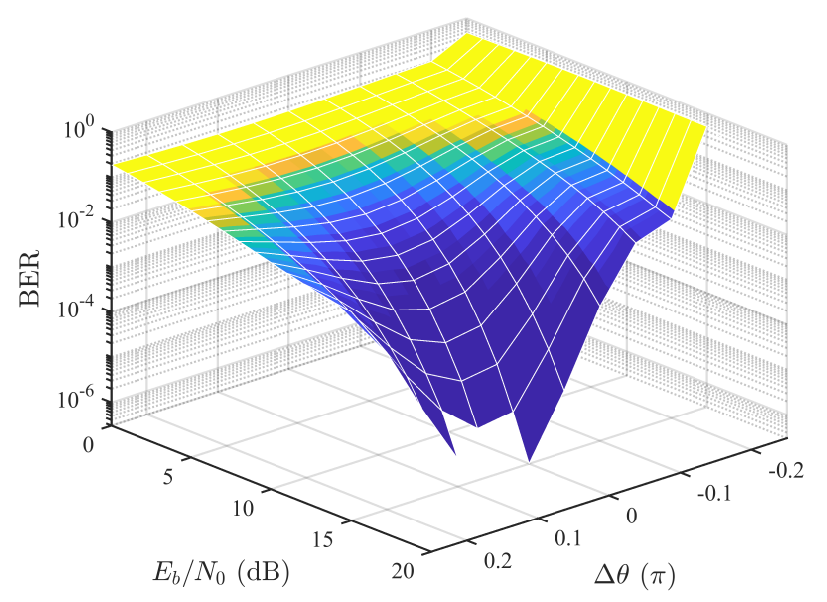

Fig. 5. BER versus phase offset $\Delta \theta$ and $E_{b} / N_{0}$ given the oversampling ratio $N_{s}=20$ for communicating over a single-path $\mathrm{THz}$ channel having a carrier frequency $f_{c}=220 \mathrm{GHz}$.

\section{A. Simulation System Parameters}

The classic raised cosine signalling pulse is utilized [35], where the transmit pulse is specified by

$$
p_{t r}(t)=\frac{\sin (\pi t / \tau)}{(\pi t / \tau)} \frac{\cos (\beta \pi t / \tau)}{1-\beta^{2} t^{2} / \tau^{2}},
$$

where $\beta$ is the roll-off factor and $\tau$ is the width of the pulse, given by $\beta=0.5$ and $\tau=0.01 \mathrm{~ns}$.

Furthermore, directional Cassegrain antennas are used and a single-path $\mathrm{THz}$ channel is considered, and the same hybrid distortion parameters shown in Section III have been adopted in this section.

\section{B. Effect of $\Delta \theta$ and $N_{s}$}

Firstly, to verify the correctness of the analysis in Subsection III-A, the simulation based bit error rate (BER) performance is presented in this subsection, where the symbols satisfying $T_{s}=2 \tau$ are generated.

In Fig. 5, we plot the BER versus the phase offset $\Delta \theta$ and $E_{b} / N_{0}$ for the oversampling ratio $N_{s}=20$. It can be seen that there exists a unique $\Delta \theta$ that minimizes the BER performance which also depends on $E_{b} / N_{0}$. This demonstrates that it is important to compensate the phase offset for improving the BER performance.

Furthermore, in Fig. 6 we characterize the impact of the phase offset on the achievable BER, given various $E_{b} / N_{0}$ values and $N_{s}=20$. Again, it can be seen that the BER is minimized when the phase offset is around $0.04 \pi$. Moreover, the simulation-based BER matches the analytical result of Subsection III-A, where the BER is reduced when the deflection ratio is increased.

In Fig. 7, we further plot the BER versus $E_{b} / N_{0}$ parameterized by $N_{s}$ at $\Delta \theta=0$. It is observed that as expected, the BER performance over AWGN channels is better that over the $\mathrm{THz}$ channel due to the hybrid distortions. We can see that even the optimal receiver fails to demodulate the singlebit sequence when $N_{s}=1$. It is also observed that the BER trend is consistent with the deflection ratio trend of Fig. 2.

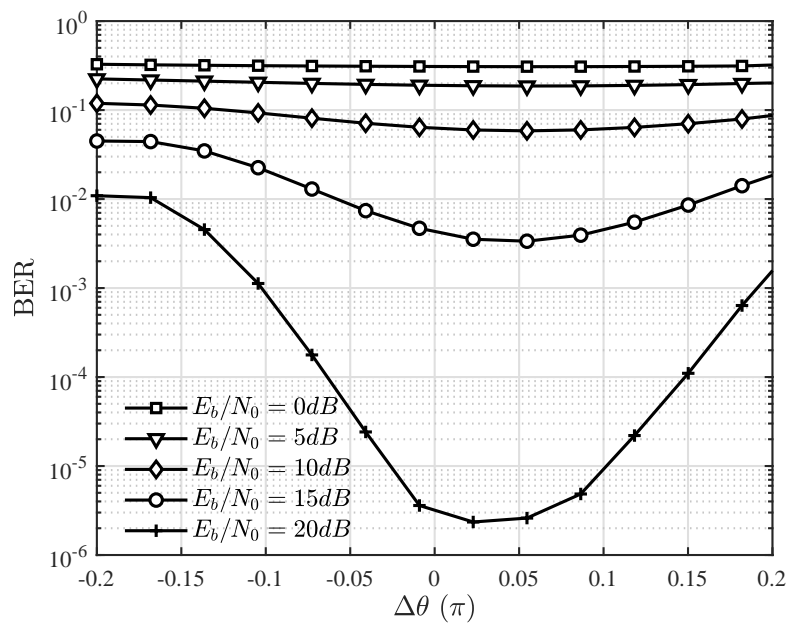

Fig. 6. BER versus phase offset $\Delta \theta$ for different $E_{b} / N_{0}$, given $N_{s}=20$.

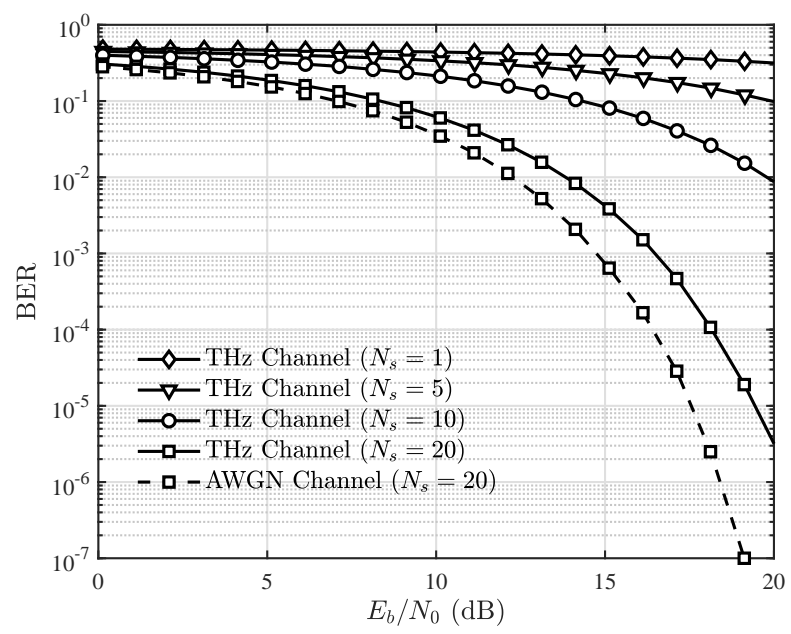

Fig. 7. BER versus $E_{b} / N_{0}$ for different $N_{s}$, given $\Delta \theta=0$.

\section{Phase Offset Difference Estimation Performance}

We now present our simulation results for validating the efficiency of our proposed algorithm detailed in Subsection III-B for estimating the phase offset difference. Throughout this subsection, the initial phase offset is set to 0 , and $E_{b} / N_{0}=$ $5 \mathrm{~dB}$. The optimal phase offset is approximately $0.04 \pi$ as shown in Fig. 2 (a). Thus the optimal phase offset difference is $\Delta^{2} \theta^{\star} \approx 0.04 \pi$.

To investigate the convergence properties of Algorithm 1, we generate $N_{\text {run }}=1000$ independent phase offset training sequences, and the length of each training sequence is $N_{p} N_{t}$, given by $N_{p}=1000$ and $N_{t}=200$. The step size is set to $\alpha_{\theta}=0.01 \pi$. The evolution of the population of the estimated phase offset differences is shown in Fig. 8. Specifically, in Fig. 8, we plot $\Delta^{2} \widetilde{\theta}_{t, n}$ versus $t$ for all the $N_{\text {run }}$ realizations, where $\Delta^{2} \widetilde{\theta}_{t, n}$ is the $t$-th estimated phase offset difference at the $t$-th iteration of the $n$-th realization. Since the number of training symbols is limited in each iteration and the estimated weight tuple $\widehat{\mathbf{w}}$ is realization dependent, the estimated phase offset difference $\Delta^{2} \widetilde{\theta}_{t, n}$ naturally fluctuates from realization 


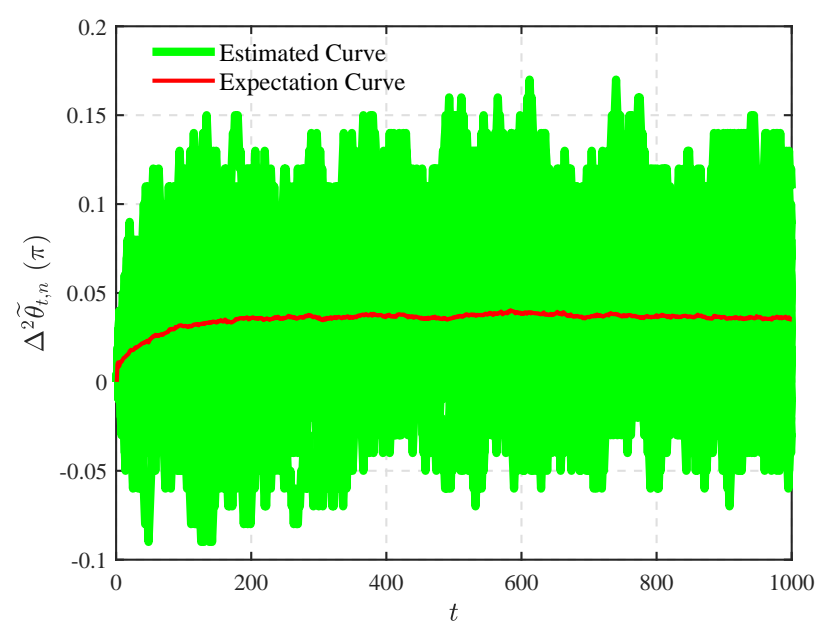

Fig. 8. Evolution of the population of the estimated differences between optimal phase offset and actual phase offset over 1000 realizations given $N_{t}=$ 200 .

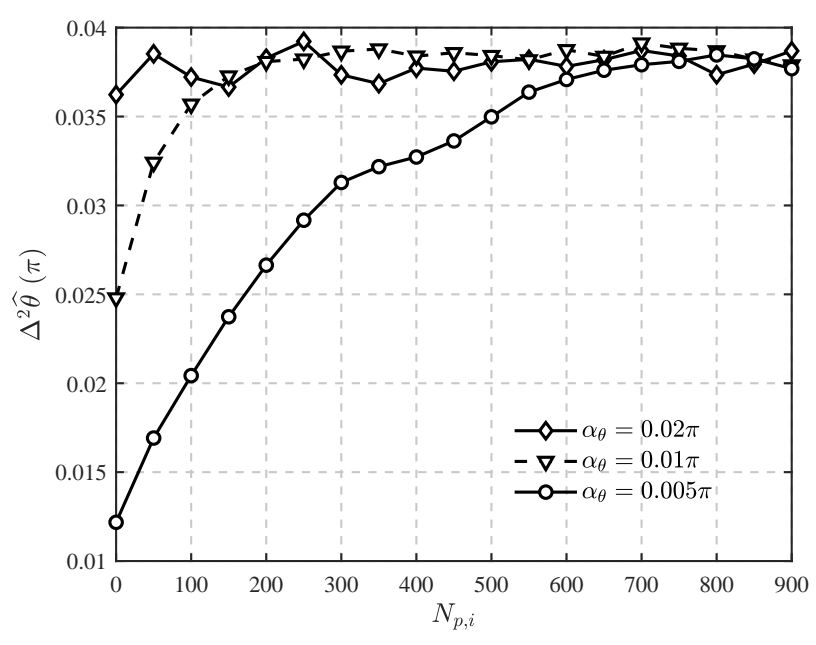

Fig. 9. The estimated $\Delta^{2} \widehat{\theta}$ versus $N_{p, i}$ parameterized by $\alpha_{\theta}$ given $N_{t}=$ 200 and $N_{p}=N_{p, i}+100$.

to realization, that is, it is stochastic. However, the expectation of $\Delta^{2} \widetilde{\theta}_{t, n}$, approximated by

$$
\Delta^{2} \bar{\theta}_{t}=\frac{1}{N_{\text {run }}} \sum_{n=1}^{N_{\text {run }}} \Delta^{2} \widetilde{\theta}_{t, n}
$$

does converge to the optimal phase offset difference $0.04 \pi$. Also observe from Fig. 8 that $\Delta^{2} \widetilde{\theta}_{t, n}$ converges after $t=150$. Recall that in Algorithm 1, the final estimated phase offset difference, denoted as $\Delta^{2} \widehat{\theta}_{n}$ here, is obtained by averaging $\Delta^{2} \widetilde{\theta}_{t, n}$ over $t=N_{p, i}$ to $N_{p}$ to reduce the fluctuation of $\Delta^{2} \widetilde{\theta}_{t, n}$. In this case, we can see that $N_{p, i}=150$ is appropriate.

Next we demonstrate that $\Delta^{2} \widehat{\theta}_{n}$ converges to the optimal phase offset difference, namely, the expectation of $\Delta^{2} \widehat{\theta}_{n}$ is the optimal phase offset difference. With $N_{t}=200$ and $N_{p}=N_{p, i}+100$, in Fig. 9 , we plot the average of $\Delta^{2} \widetilde{\theta}_{n}$ over the 1000 realizations, denoted as $\Delta^{2} \widehat{\theta}$ here, as the function of $N_{p, i}$ parameterized by $\alpha_{\theta}$. It is observed that as the step size $\alpha_{\theta}$ increases, the number of the subsequences $N_{p, i}$ for $\Delta^{2} \widehat{\theta}$ to approach the optimal value decreases, thus decreasing the

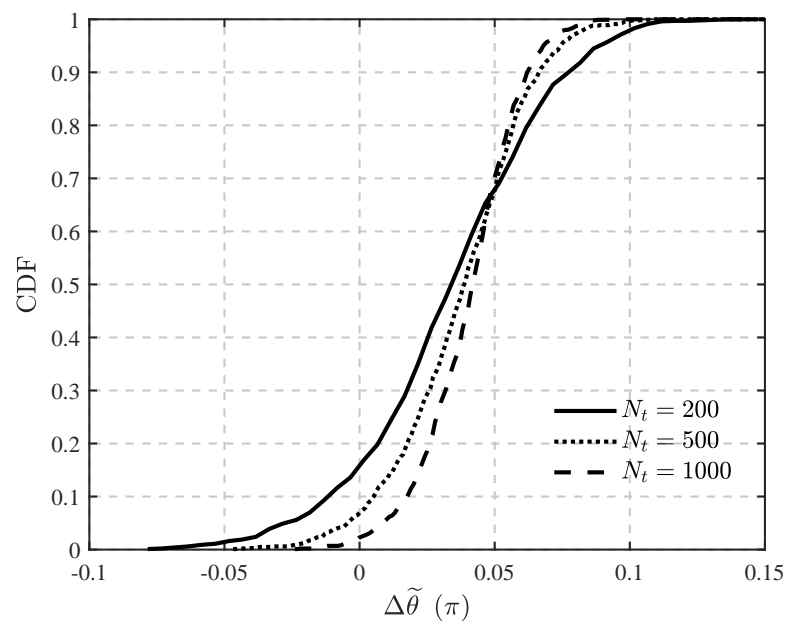

Fig. 10. The CDF of the estimated $\Delta^{2} \widehat{\theta}$ parameterized by $N_{t}$ given $N_{p}=$ 250 and $N_{p, i}=150$.

training overhead. However, the estimated $\Delta^{2} \widehat{\theta}$ will fluctuate more seriously as the step size $\alpha_{\theta}$ increases, which will limit the accuracy of the estimated $\Delta^{2} \widehat{\theta}$. Hence, it is important to select an appropriate step size to realize an overhead versus performance trade-off. In this case, we can see that it is appropriate to utilize the average value over the last $N_{p}-N_{p, i}=100$ subsequences as the estimate of phase offset difference with the step size set to $\alpha_{\theta}=0.01 \pi$.

In Fig. 10, we plot the cumulative distribution function (CDF) of the estimated phase offset difference obtained by Algorithm 1, parameterized by $N_{t}$ and given $N_{p}=250$ and $N_{p, i}=150$. Observe that the estimated $\Delta^{2} \widehat{\theta}$ is concentrated around $0.04 \pi$, again indicating the effectiveness of Algorithm 1. Also as expected, as the length $N_{t}$ of subsequence increases, leading to more accurate estimate, the CDF curve becomes steeper, that is, the probability that the estimated phase offset difference is around $0.04 \pi$ becomes higher.

Based on the above investigations as well as considering training overhead and performance trade-off, $N_{t}=200$, $N_{p}=250, N_{p, i}=150$ and $\alpha_{\theta}=0.01 \pi$ are selected as the algorithmic parameters of Algorithm 1 to compensate the phase offset for the following demodulation operation. With this set of algorithmic parameters, the training overhead or the length of PEF field is $N_{p} N_{t}=50000$ symbols, which is acceptable considering huge throughput of $\mathrm{THz}$ systems, while ensuring a sufficiently accurate phase compensation performance.

\section{Demodulation Performance}

Let us now examine the detection performance of our proposed learning based DFNN scheme against that of the optimal and suboptimal demodulation schemes of [17], and the eight-sector phase based scheme in [18]. In the simulations, we note that 50,000 training symbols are sufficient for training the weights of the optimal and suboptimal receiver [17] and the eight-sector phase based receiver of [18] for it to reach its full performance potential. Hence, increasing the number of training symbols further does not improve the achievable 


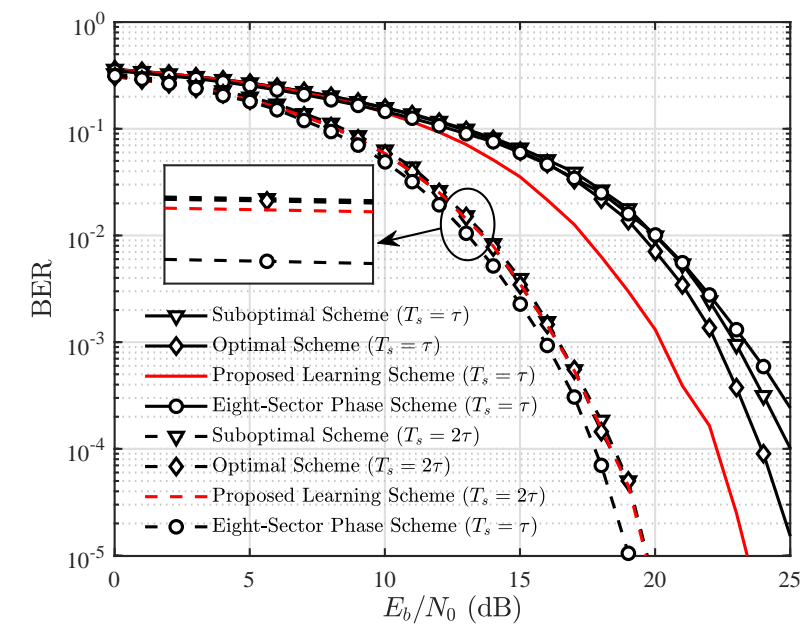

Fig. 11. Performance comparison of the proposed learning-based DFNN demodulation scheme, the optimal demodulation scheme of [17], the suboptimal demodulation scheme of [17] and the eight-sector phase scheme of [18] for $T_{s}=\tau$ and $T_{s}=2 \tau$.

performance. For a fair comparison, we also train our DFNN with $N_{d}=50,000$ training symbols $^{3}$. Note that a large number of samples is required for the empirical cross entropy (35) to approach the ensemble cross entropy (34), because the underlying distribution of the demodulation process is highly nonlinear and non-Gaussian. Hence it is advisable to use a sufficiently long DTF field, and $N_{d}=50,000$ is suitable. A DFNN having $L=4$ hidden layers is utilized for demodulating the received single-bit sequence, and the numbers of neurons in the four hidden layers are $(4,4,4,4)$, respectively. The total number of adaptive parameters for this DFNN is $N_{\text {tot }}=244$, given the oversampling ratio of $N_{s}=20$.

In Fig. 11, we plot the BER as a function of $E_{b} / N_{0}$ associated with $N_{s}=20$ and $\Delta \theta=0$ for the four schemes compared. It can be seen from Fig. 11 that all the schemes perform equally well when $T_{s}=2 \tau$, and the BER curves of the optimal schemes, suboptimal scheme and our proposed learning-based scheme are indistinguishable. Upon relying on two extra analog branches, namely the I+Q and I-Q branches, the BER performance of the eight-sector phase based receiver [18] improves slightly, explicitly a $0.5 \mathrm{~dB}$ performance gain can be obtained at the BER level of $10^{-2}$. However, the receiver architecture of the eight-sector phase based scheme becomes more complex compared to the other three schemes, which requires two extra analog adders and single-bit ADCs.

When $T_{s}=\tau$, however, the performance of the four schemes degrade significantly. This is because the adjacent symbols are no longer independent for $T_{s}=\tau$ and the correlation between the symbols cannot be ignored. As a result, the distribution of each sampling point will be different from the estimated distribution obtained from the training samples. Note that the

\footnotetext{
${ }^{3}$ Based on [36] and [37], the correlation of phase noise can be guaranteed when the frame length is smaller than $\ln (2) /\left(2 \pi^{2} T_{s}^{2} f_{0}^{2}\right)$, where $f_{0}$ is the corner frequency of the oscillator. Considering the oscillator of [38], the maximum frame length satisfying the correlation of phase noise may be as high as $3.5 \times 10^{6}$, which is higher than $N_{p} N_{t}+N_{d}$. Therefore, the efficiency of our training solution can be guaranteed.
}

performance of the optimal scheme [17], of the suboptimal scheme [17] and of the eight-sector phase based scheme [18] become particularly limited for $T_{s}=\tau$. By contrast, upon considering the change in distribution caused by the correlation between symbols our learning based DFNN detection scheme demodulates the single-bit sequence more accurately without relying on the distribution of each sample. Specifically, at the BER level of $10^{-2}$, our DFNN demodulation scheme offers more than $2.5 \mathrm{~dB} E_{b} / N_{0}$ gain over both the optimal and the suboptimal schemes of [17].

\section{CONCLUSIONS}

In this paper, a deep learning-assisted demodulation scheme has been proposed for single-bit THz QPSK receivers, which consists of an estimator for the difference between the optimal phase offset and the actual phase offset, a phase offset compensator, two single-bit ADCs, and a deep learning based demodulation network. Based on the deflection ratio of the maximum-likelihood detector, which is the optimal detector for the single-bit receiver, we have investigated the impact of phase offset on the demodulation performance. This has led us to develop an estimator for the phase offset difference in order to compensate the phase offset accordingly before sampling. A deep feedforward neural network has been proposed to demodulate the phase offset compensated single-bit sampled sequence. Moreover, a twin-phase training strategy has been proposed for training the phase offset difference estimator and the demodulation DFNN. The simulation results have demonstrated that the proposed demodulation scheme is capable of improving the BER of the $\mathrm{THz}$ receiver under single-bit quantization and severe hybrid distortions.

\section{APPENDIX A \\ PROOF OF (26).}

Relying on the distribution of the sampling points, which follows the Gaussian distribution due to the additive Gaussian noise, the probability of each sampling point belonging to \pm 1 when the decision results of the information bits $\left(d_{k 1}, d_{k 0}\right)$ are $(1,1)$ can be expressed as

$$
\begin{aligned}
& \operatorname{Pr}\left(r_{I, n}=1 \mid d_{k 1}=1, d_{k 0}=1\right)=1-Q\left(\widetilde{\gamma}_{I, n}^{1,1}\right), \\
& \operatorname{Pr}\left(r_{I, n}=-1 \mid d_{k 1}=1, d_{k 0}=1\right)=Q\left(\widetilde{\gamma}_{I, n}^{1,1}\right), \\
& \operatorname{Pr}\left(r_{Q, n}=1 \mid d_{k 1}=1, d_{k 0}=1\right)=1-Q\left(\widetilde{\gamma}_{Q, n}^{1,1}\right), \\
& \operatorname{Pr}\left(r_{Q, n}=-1 \mid d_{k 1}=1, d_{k 0}=1\right)=Q\left(\widetilde{\gamma}_{Q, n}^{1,1}\right) .
\end{aligned}
$$

Furthermore, based on (20), the expectation of the decision result can be calculated as

$$
\begin{aligned}
& \mathbb{E}\left(\widetilde{\lambda}_{k} \mid d_{k 1}=1, d_{k 0}=1\right) \\
& =\sum_{n=1}^{N_{s}}\left(\log \left(2-2 Q\left(\widetilde{\gamma}_{I, n}^{1,1}\right)\right)\left(1-Q\left(\widetilde{\gamma}_{I, n}^{1,1}\right)\right)\right. \\
& \quad+\log \left(2 Q\left(\widetilde{\gamma}_{I, n}^{1,1}\right)\right) Q\left(\widetilde{\gamma}_{I, n}^{1,1}\right) \\
& \quad+\log \left(2-2 Q\left(\widetilde{\gamma}_{Q, n}^{1,1}\right)\right)\left(1-Q\left(\widetilde{\gamma}_{Q, n}^{1,1}\right)\right) \\
& \left.\quad+\log \left(2 Q\left(\widetilde{\gamma}_{Q, n}^{1,1}\right)\right) Q\left(\widetilde{\gamma}_{Q, n}^{1,1}\right)\right)-2 N_{s} \log (2) .
\end{aligned}
$$


Similar, $\mathbb{E}\left(\widetilde{\lambda}_{k} \mid d_{k 1}=1, d_{k 0}=0\right)$ can be calculated as

$$
\begin{aligned}
& \mathbb{E}\left(\widetilde{\lambda}_{k} \mid d_{k 1}=1, d_{k 0}=0\right) \\
& =\sum_{n=1}^{N_{s}}\left(\log \left(2-2 Q\left(\widetilde{\gamma}_{I, n}^{1,0}\right)\right)\left(1-Q\left(\widetilde{\gamma}_{I, n}^{1,1}\right)\right)\right. \\
& \quad+\log \left(2 Q\left(\widetilde{\gamma}_{I, n}^{1,0}\right)\right) Q\left(\widetilde{\gamma}_{I, n}^{1,1}\right) \\
& \quad+\log \left(2 Q\left(\widetilde{\gamma}_{Q, n}^{1,1}\right)\right)\left(1-Q\left(\widetilde{\gamma}_{Q, n}^{1,1}\right)\right) \\
& \left.\quad+\log \left(2 Q\left(\widetilde{\gamma}_{Q, n}^{1,0}\right)\right) Q\left(\widetilde{\gamma}_{Q, n}^{1,1}\right)\right)-2 N_{s} \log (2) .
\end{aligned}
$$

Substituting (39) and (40) into (25), (26) can be obtained.

\section{REFERENCES}

[1] I. F. Akyildiz, J. M. Jornet, and C. Han, "TeraNets: Ultra-broadband communication networks in the Terahertz band," IEEE Wireless Commun., vol. 21, no. 4, pp. 130-135, Aug. 2014.

[2] A.-A. A. Boulogeorgos, E. N. Papasotiriou, and A. Alexiou, "Analytical performance assessment of THz wireless systems," IEEE Access., vol. 7, pp. 11436-11453, Jan. 2019.

[3] K. Huang and Z. Wang, "Terahertz Terabit wireless communication," IEEE Microwave Mag., vol. 12, no. 4, pp. 108-116, Jun. 2011.

[4] A. Alexiou, S. Andreev, G. Fodor, and T. Nagatsuma, "THz communications: A catalyst for the wireless future," IEEE Commun. Mag., vol. 58, no. 11, pp. 12-13, Nov. 2020.

[5] L. Zhang, X. Pang, S. Jia, S. Wang, and X. Yu, "Beyond $100 \mathrm{~Gb} / \mathrm{s}$ optoelectronic Terahertz communications: Key technologies and directions," IEEE Commun. Mag., vol. 58, no. 11, pp. 34-40, Nov. 2020.

[6] B. Aazhang, et al., "Key drivers and research challenges for 6G ubiquitous wireless intelligence," White Paper, 09 2019. [Online]. Available: http://jultika.oulu.fi/files/isbn9789526223544.pdf

[7] A.-A. A. Boulogeorgos, et al., "Wireless Terahertz system architectures for networks beyond 5G," Terranova Consortium, White Paper 1.0, Jul. 2018. [Online]. Available: https://arxiv.org/ftp/arxiv/papers/1810/1810.12260.pdf

[8] Y. R. Ramadan, H. Minn, and M. E. Abdelgelil, "Precompensation and system parameters estimation for low-cost nonlinear Tera-Hertz transmitters in the presence of I/Q imbalance," IEEE Access, vol. 6, pp. 51814-51833, 2018.

[9] T. Mao, Q. Wang, and Z. Wang, "Spatial modulation for Terahertz communication systems with hardware impairments," IEEE Trans. Vehi. Technol., vol. 69, no. 4, pp. 4553-4557, Apr. 2020.

[10] T. Schenk, RF Imperfections in High-Rate Wireless Systems: Impact and Digital Compensation. The Netherlands: Springer, 2008.

[11] Y. Chiu, B. Nikolic, and P. Gray, "Scaling of analog-to-digital converters into ultra-deep-submicron CMOS," in Proc. 2005 IEEE Custom Integrated Circuits Conf. (San Jose, CA, USA), Sep. 21, 2005, pp. 375-382.

[12] P. Neuhaus, et al., "Sub-THz wideband system employing 1-bit quantization and temporal oversampling," in Proc. ICC 2020 (Dublin, Ireland), Jun. 7-11, 2020, pp. 1-7.

[13] F. Wang, J. Fang, H. Li, Z. Chen, and S. Li, "One-bit quantization design and channel estimation for massive MIMO systems," IEEE Trans. Vehi. Technol., vol. 67, no. 11, pp. 10921-10934, Nov. 2018.

[14] F. Liu, et al., "Angular-domain channel estimation for one-bit massive MIMO systems: Performance bounds and algorithms," IEEE Trans. Vehi. Technol., vol. 69, no. 3, pp. 2928-2942, Mar. 2020.

[15] S. Hoyos, B. Sadler, and G. Arce, "Monobit digital receivers for ultra wideband communications," IEEE Trans. Wireless Commun., vol. 4, no. 4, pp. 1337-1344, Jul. 2005.

[16] H. Yin, Z. Wang, L. Ke, and J. Wang, "Monobit digital receivers: Design, performance, and application to impulse radio," IEEE Trans. Commun., vol. 58, no. 6, pp. 1695-1704, Jun. 2010.

[17] Z. Wang, H. Yin, W. Zhang, and G. Wei, "Monobit digital receivers for QPSK: Design, performance and impact of IQ imbalances," IEEE Trans. Commun., vol. 61, no. 8, pp. 3292-3303, Aug. 2013.

[18] J. Singh and U. Madhow, "Phase-quantized block noncoherent communication," IEEE Trans. Commun., vol. 61, no. 7, pp. 2828-2839, Jul. 2013.

[19] A. Wadhwa and U. Madhow, "Blind phase/frequency synchronization with low-precision ADC: A Bayesian approach," in Proc. ALLERTON, Oct. 2013, pp. 181-188.
[20] A. Wadhwa and U. Madhow, "Near-coherent QPSK performance with coarse phase quantization: A feedback-based architecture for joint phase/frequency synchronization and demodulation," IEEE Trans. Signal Process., vol. 64, no. 17, pp. 4432-4443, Sep. 2016.

[21] T. O Shea and J. Hoydis, "An introduction to deep learning for the physical layer," IEEE Trans. Cogn. Commun. and Netw., vol. 3, no. 4, pp. 563-575, Dec. 2017.

[22] E. Balevi and J. G. Andrews, "One-bit OFDM receivers via deep learning," IEEE Trans. Commun., vol. 67, no. 6, pp. 4326-4336, Jun. 2019.

[23] E. Balevi and J. G. Andrews, "Autoencoder-based error correction coding for one-bit quantization," IEEE Trans. Commun., vol. 68, no. 6, pp. 3440-3451, Jun. 2020.

[24] Y. Jeon, N. Lee, and H. V. Poor, "Robust data detection for MIMO systems with one-bit ADCs: A reinforcement learning approach," IEEE Trans. Wireless Commun., vol. 19, no. 3, pp. 1663-1676, Mar. 2020.

[25] Y. Zhang, M. Alrabeiah, and A. Alkhateeb, "Deep learning for massive MIMO with 1-bit ADCs: When more antennas need fewer pilots," IEEE Wireless Commun. Lett., vol. 9, no. 8, pp. 1273-1277, Aug. 2020.

[26] I. Goodfellow, Y. Bengio, and A. Courville, Deep Learning. Cambridge, MA, USA: MIT Press, 2016.

[27] C. Han and Y. Chen, "Propagation modeling for wireless communications in the Terahertz band," IEEE Commun. Mag., vol. 56, no. 6, pp. 96-101, Jun. 2018.

[28] A.-A. A. Boulogeorgos, E. N. Papasotiriou, and A. Alexiou, "Analytical performance evaluation of $\mathrm{THz}$ wireless fiber extenders," in Proc. PIMRC 2019 (Istanbul, Turkey), Sep. 8-11, 2019, pp. 1-6.

[29] H. Cao, A. S. Tehrani, C. Fager, T. Eriksson, and H. Zirath, "I/Q imbalance compensation using a nonlinear modeling approach," IEEE Trans. Microwave Theory Tech., vol. 57, no. 3, pp. 513-518, Mar. 2009.

[30] G. Colavolpe, A. Barbieri, and G. Caire, "Algorithms for iterative decoding in the presence of strong phase noise,'IEEE J. Sel. Areas Commun., vol. 23, no. 9, pp. 1748-1757, Sep. 2005.

[31] B. Picinbono, "On deflection as a performance criterion in detection," IEEE Trans. Aerospace and Electron. Systems, vol. 31, no. 3, pp. 1072 1081, Jul. 1995.

[32] H. Grubinger, G. von Buren, H. Barth, and R. Vahldieck, "Continuous tunable phase shifter based on injection locked local oscillators at 30 GHz," in 2006 IEEE MTT-S Int. Microw. Symp. Dig. (San Francisco, CA, USA), Jun. 11-16, 2006, pp. 1821-1824.

[33] M. F. Møller, "A scaled conjugate gradient algorithm for fast supervised learning,"Neural Netw., vol. 6, no. 4, pp. 525-533, Nov. 1993.

[34] C. M. Bishop, Neural Networks for Pattern Recognition. UK: Oxford University Press, 1996.

[35] U. Madhow, Fundamentals of Digital Communication. UK: Cambridge University Press, 2008.

[36] N. Bouhlel, M. Saad and F. Bader, "Sub-Terahertz wireless system using dual-polarized generalized spatial modulation with RF impairments," IEEE J. Sel. Areas Commun., vol. 39, no. 6, pp. 1636-1650, Jun. 2021.

[37] S. Bicais and J. Dore, "Design of digital communications for strong phase noise channels," IEEE Open J. Veh. Technol., vol. 1, pp. 227243, 2020.

[38] S. Li, D. Fritsche, C. Carta, and F. Ellinger, "A 200-GHz sub-harmonic injection-locked oscillator with $0-\mathrm{dBm}$ output power and 3.5 DC-toRFefficiency," in Proc. IEEE Radio Freq. Integr. Circuits Symp., Jun. 2018, pp. 212-215. 